\title{
C-3C: a structure for high reliability with minimal redundancy for batteries
}

\author{
Christophe SAVARD ${ }^{(1),(2)}$ \\ Laboratoire Ampère UMR CNRS 5005 \\ Université de Lyon \\ (1) Université Claude Bernard Lyon - 43 bd du 11 \\ novembre 1918 - 69622 Villeurbanne Cedex / France \\ (2) INSA de Lyon - 20 avenue Albert Einstein - \\ 9621 Villeurbanne Cedex / France
}

\author{
Ali SARI, Pascal VENET, \\ Laboratoire Ampère UMR CNRS 5005 \\ (1) Université Claude Bernard de Lyon \\ Laurent PIETRAC, Eric NIEL \\ Laboratoire Ampère UMR CNRS 5005 \\ (2) INSA de Lyon
}

\begin{abstract}
This paper deals with the structures involving the storage cells of electrical energy and on the reconfiguration of these storage systems. It places the existing structures in a matrix configuration to compare their performances in terms of reliability. Then, a different solution: the $\mathrm{C}-3 \mathrm{C}$ structure, for limiting the redundancy for fault tolerance of the cells is proposed and compared with existing patterns. This new configuration, besides it minimizes redundancy, improves battery reliability and increase reliability of a matrix structure of cells.
\end{abstract}

Keywords - balancing, reconfiguration, redundancy, reliability batteries.

\section{INTRODUCTION}

Often, electrical energy storage cells, whatever their technologies, does not have a sufficient voltage and current level to respect the expectations of the entity that wishes to use it as power supply. Then, the batteries consist of an association of a large number of elementary cells. Also, the cells are associated in series to increase the output voltage of the battery. There are associated in parallel to increase the current supplied. In order to modulate the output voltage and current, it may be necessary to be able to connect all or a part of cells inserted in the structure. This function is provide by switches.

To order these switches, a Battery Management System (BMS) is used. It defines which cells must be activated and which ones should be at rest. To do this, it is based on global data collected within the battery and on the data of each element. In presence of redundant cells, one of his goals for the managed elements consists in preserving the cells based on their State of Charge (SoC). Nevertheless, the association of cells penalizes the reliability of the device. Take the hypothesis that the reliability of the switches is clearly larger than cells. This allows us to admit in a structure associating cells and switches the reliability of the cell as dominant parameter. If we associate $n$ cells in series in order to deliver a voltage of $n$ times the voltage of a single cell, the reliability of the system is much less than that a single cell since the reliability is equal to the cell reliability in the $n$th power.
Without redundancy, all cells must be in working order. If a cell is in default, the system goes into default. To increase fault tolerance, it is commonly added redundant cells that will make up the failing cells. Depending on the architecture of the batteries, the overall reliability is improved more or less depending on the redundancy rate. Also, it may be convenient to define a structure capable of improving reliability without unduly increasing the number of redundant cells.

After introducing the subject, the second part of this article deals with equalization, recalling that this principle ensures greater availability of energy stored in cells associated within a battery. The section III refers to some examples of structures from literature for involving cells in multicellular systems. Before discussing in point $\mathrm{V}$ our proposal of architecture, we specify in item IV the way we associate the cells within the battery. Section VI classifies the various solutions proposed.

\section{BALANCING AND RECONFIGURATION}

As each element can't inherently be exactly the same to another (because of tolerances in manufacturing, of different connections) and as it is not routinely used in the same conditions (leading to different environment and aging), each not present same characteristic at $t$ time: voltage, State of Health (SoH) [1], series resistance, temperature... To lessen the consequences of these disparities between items, BMS and equalizing devices then ensures balance between cells.

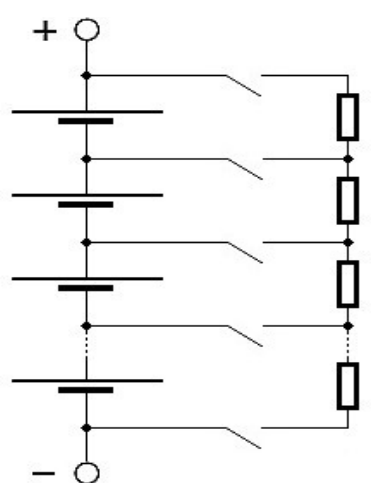

Fig. 1. Principle of dissipative balancing 
The easiest solution to implement and the most frequently used is to balance the load levels from the bottom: bring down all the elements to balance the potential (or SoC) the lowest of them $[2,3,4,5]$ by dissipation the excess energy, as showed in Fig. 1. To reduce the Joules losses, other solutions exist, as distributive balancing using passive reactance elements instead of resistors.

In this article, we consider the theoretical case where a cell delivers a current unit under a voltage unit, and we'll assume that all cells are identical.

In a battery, or in a part of the battery, it is necessary to balance the elements to allow a good operability of the stored energy. In fact, consider a hypothetical example in Fig. 2a) of three cells connected in series to increase the voltage delivered. Consider the $\mathrm{SoC}$ as the parameter to be balanced, the latter ranging from $100 \%$ to $0 \%$ of the usable capacity of a cell. If we have three disparately storing elements, a certain amount of energy can't be recovered. The battery will cease to be functional when the lowest cell will be empty. If we balance the states of charges, we can expect recover more.

a)
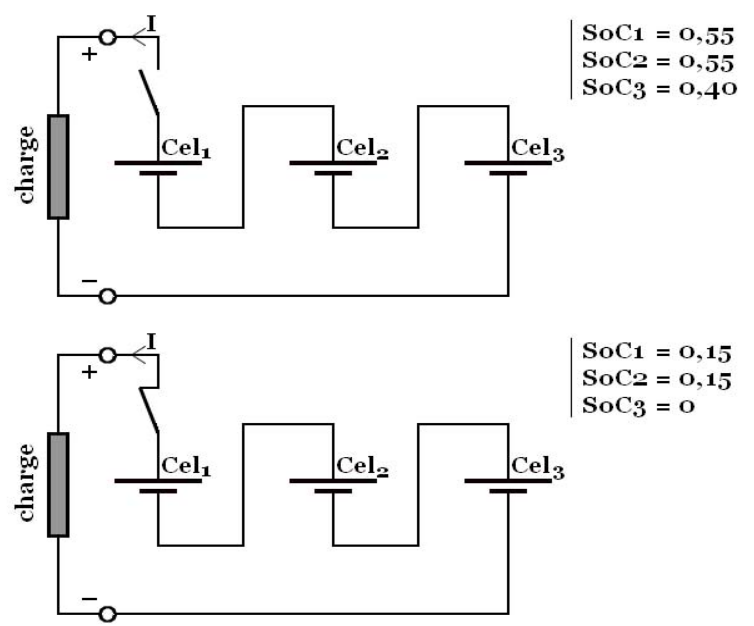

b)
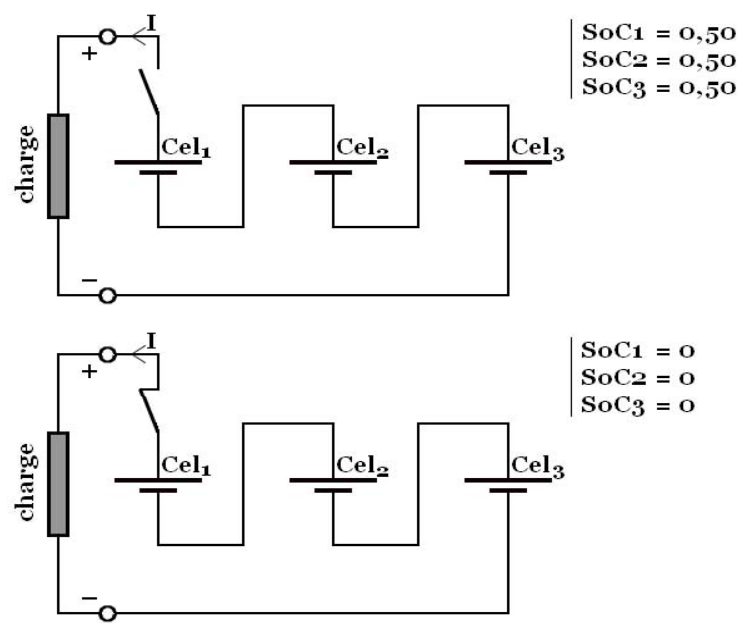

Fig. 2. Interest of balancing. a) Before balancing, in this example, with the various SoC on each cell in series, we can only extract $80 \%$ of the storaged energy. b) After balancing, with identical sum of SoC, all the stored energy can be extracted, in the present example
This is the case in Fig. 2b), where we have the same amount of energy, but distributed evenly between the connected elements. As the three cells in this example running out simultaneously, we can recover all the stored energy. If, moreover, the balancing between the SoC cells has not been made with dissipative matter but distributive matter, via reactance components or directly between cells, the energetic efficiency is to the advantage of balancing.

Balancing is therefore essential to improved efficiency of the storage systems. This balancing requires the use a number of switches.

On the other hand, if you desired to preserve a portion of the internal resources of the battery, espacially when a cell is approching a deep discharge, it is necessary to be able to isolate the cell concerned, by disconnecting it to its neighbors whatever the architecture: series or parallel. This isolation can be realized by the use of switches.

Finally, in order to overcome the failure of cells, and then use longer battery by tolerating internal faults, it is necessary to resort to redundancy in terms of number of cells: having a number of cells in the structure higher than it is necessary to meet the specifications (voltage and current to deliver). Obviously, this redundancy increases the manufacturing cost and overhead of arbitration between redundancy and improved service life is achieved in order to define the optimal size of this redundancy. The switches mentioned above, securing insulation cells, are highly requisite to improve battery performance. BMS utilize its to remove from its list all those resources in default

From this, we can draw a line of research: why not use the same switches for ensuring balancing and for allowing a reconfiguration of the internal structure of the battery? [6]

\section{STATE OF THE ART}

Batteries generally consist of assemblies of modules which are connected in series and in parallel. There are many internal configurations to these modules. The structures range from the simplest to more complicated structures. Typically the number of switches (actually transistors) associated with the cells increases with the complexity. In these lines, we will just mention a few examples.

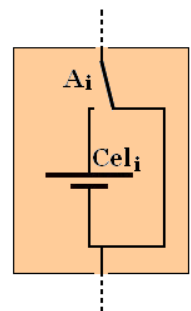

Fig. 3. Easiest circuit for a module, combining a cell and a double switch

The simplest structure consists to associate a cell with a double switch as made in Fig. 3. In this case, the module combining the cell to the switch may either issue a current unit with a voltage unit or behave as a closed circuit [7]. It is normally used in series. 
When the cell is defective, it is shunted by the switch, without being put it in short-circuit, allowing to use it subsequently when charge is completed (in the case the cell undergoes an applicable failure in discharging). By adding redundant modules, the structure can be repeated identically. Suppose we run in maturity phase, where the failure rate is constant and reliability follows an exponential law. If the application needs $n$ cells in series, the Mean Time To Failure (MTTF) of the battery is equal to the MTTF of a cell divided by $n$, as recalled by the formula 1 .

By associating $n$ cells of Figure 3, we obtain:

$$
M T T F_{\text {battery }}=\frac{M T T F_{\text {cell }}}{n}
$$

Anyway, this structure only allows increasing the output voltage of the battery.

The structure of Fig.4 shows the connection structure Desa developed in [8]. It comprises three switches associated with the cell. In this way, for the outer mounting, the module (in this case the association of a cell and switches) can deliver the voltage of the cell between two of its ports, behave as a short circuit or as an open circuit.

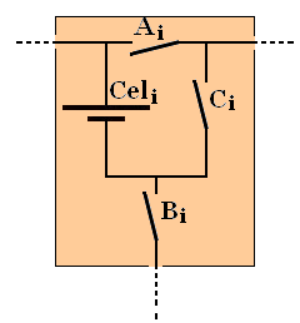

Fig. 4. DESA structure, associating a cell and three switches and increasing the number of possible configurations in the structure

The structure is advertised as improving reliability, especially in achieving this increase in the order of $50 \%$ with a redundancy of the same order. It has difficulty to be implanted since it requires long calculation times and does not handle batteries including too many cells.

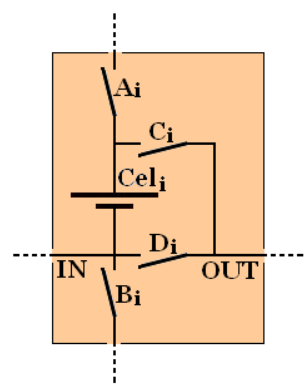

Fig. 5 Module used in a power tree, allowing any useful configurations

Finally, by combining the cell with four switches, all the useful settings for outdoor use involving a large number of modules can be possible between the four terminals of the module [9]. An example from montage principles, as power tree [10], is given in Fig. 5.
This structure allows all connection: serial, parallel, transparent (short circuit in serial mode, open circuit in parallel mode). Since the module includes four switches, which must operate on two quadrants (the current changes direction depending on whether the cell is charging or discharging), they are each composed of two transistors mounted in anti-parallel. This increases costs and requires that commutations are perfect.

\section{COMBINATION OF MODULES}

All modules as described in the previous section can be combined in a matrix of batteries. Consider that each module is a quadrupole: + and - terminals to output the voltage and IN and OUT terminals used in the case of a series connection of the elements, showed in Fig.6a). Beyond these basic connections, all connections must be possible within this quadrupole to connect the cell onto terminals. This structure which can receive these modules assembled in series as in parallel allow in Fig.6b) all possible configurations, via the switches included in the modules. Indeed, to be able to associate the basic modules described above and are sure all the degrees of freedom, it is often necessary to add to the basic structure one or more switches. The configurations are realized in series connection, parallel connection, short circuit or open circuit; note that these two last modes are transparent modes respectively in series and in parallel. To avoid edge effects realized by a partial isolation of the first and last module in each line, note connected to as many cells as the others, the OUT connection of the last cell of a row must be wired to the IN connection of the first cell.

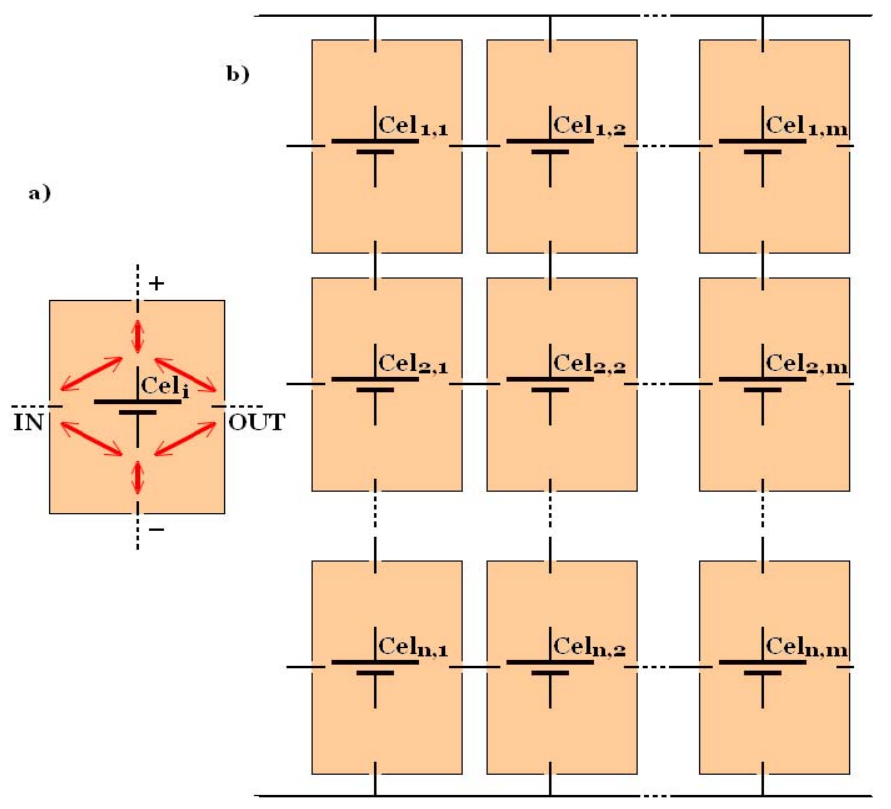

Fig. 6. a) A quadrupole structure containing a cell $i$ with its four terminals,+- , IN and OUT for all degrees of freedom: serial, parallel, and transparent for both modes. b) Matrix structure combining these quadrupole

Then schemes studied above, in Fig. 3, 4 and 5, change as shown in Fig.7. For its parts, the last diagram remains unchanged. To use Fig.3 diagram in parallel, it is necessary to cut an internal line. 
The assumptions we use in our study are: all the cells are identical, cell reliability rate is constant and well below the switches reliability rate; the switch are ideals.

\section{C-3C SOLUTION}

A matrix system to connect together the cells constituting a battery is fully relevant for two main reasons:

- Allowing a large tolerance to mistakes, by being able to eliminate any faulty cell in the matrix, whether through a permanent failure or a transient failure (battery level decrease to the deep discharge limit);

- $\quad$ Ensuring a minimum of structural redundancy.

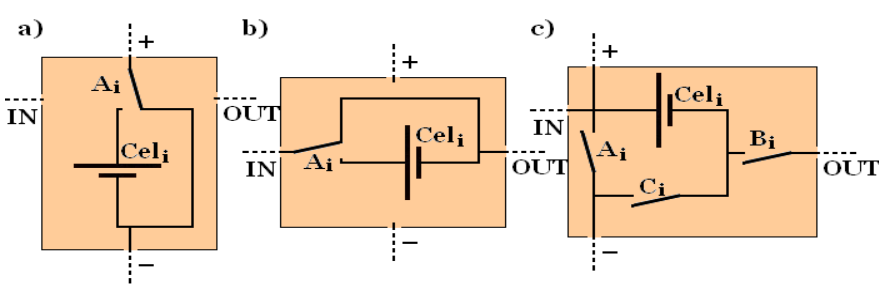

Fig. 7. a) Structure of Fig. 3 adapted for mounting in parallel.

b) Structure of Fig. 3 in a series arrangement. c) Structure of Fig. 4 adapted

Indeed, a major drawback of fault tolerant structures consists of the additional cost incurred by the redundancy. In the typical case of the matrix structure, it is possible to define a minimum of redundancy. Start from the trivial example in Fig.8a): a matrix with two rows and two columns, for supplying a current of two units at a voltage of two units (one unit per cell for current and for voltage). In such structure, if one of cell is considered defective (permanently or temporarily), as in Fig.8b), the matrix can no longer meet the demanding specifications either current or voltage.

In Fig.8c), if a redundant column is added, the matrix meets to the specifications even while there is one failed cell or less in each row. If, as in Fig.8d) two cells are in fail on the same row, the redundancy is not sufficient to meet the specifications. Thus, the addition of a column for debiting the required current insures a minimum redundancy. This reflection may be conducted regardless of the number of columns. Patently, the more the number of useful column is, the less the addition of a column will improve the life of the device.

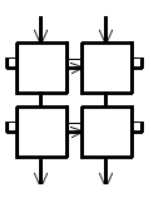

a)

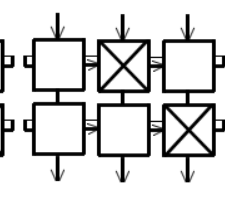

c)

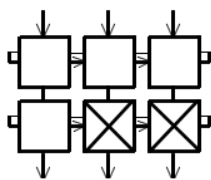

d)

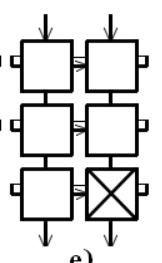

e)
Fig. 8 a) A trivial structure, with two rows and two columns. b) The failure of a single cell leads to that of the matrix. c) and d) Try the interest to add a column in aim prevent the matrix failure e) Test the interest to add a row

In Fig.8e), let's return to the trivial structure and add a row. In this case, the matrix is in default if any of cells fails. The addition of a line does not absolving the need to have as many paths to pass current as columns in the matrix. An additional cell in series into a column could maintain the terminal voltage of the battery but not respond to the current demand To conclude, the minimum redundancy for a matrix system can be defined as the addition of one column, in our application.

Extrapolating any size matrix, if we translate the flow of current in the structure into desire paths, we can see that from a cell in the row $i$ and column $j$, the current must be able to diffuse into the cells in row $i+1$, in one or other of the cells of columns $j-1, j$ and $j+1$. Hence, the scheme needs given in Fig.9.

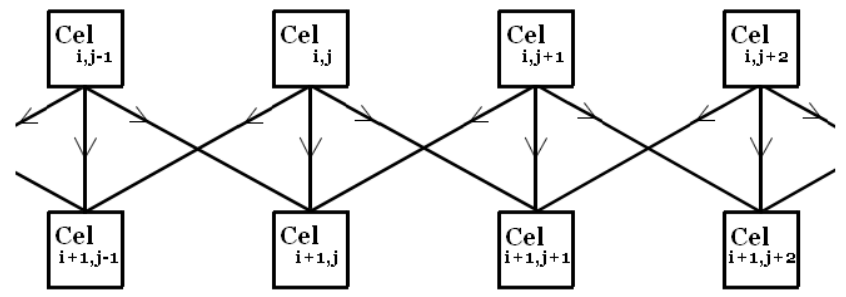

Fig. 9. Tranformation from structural sketch to graph reflectiong the derise paths

Thus, the matrix diagram in Fig.6b) is not suited to meet our desire to connect the element $\mathrm{p}_{\mathrm{ij}}$ with the three adjacent elements of the following row. Rather than seeking to connect six-pin modules, it is more appropriate to adjust the connections between modules by transforming our quadrupole to obtain a positive terminal and three negative terminals $-:-\mathrm{j}-1$, $-\mathrm{j},-\mathrm{j}_{+1}$. In terms of energy extract from the matrix, it is the same.

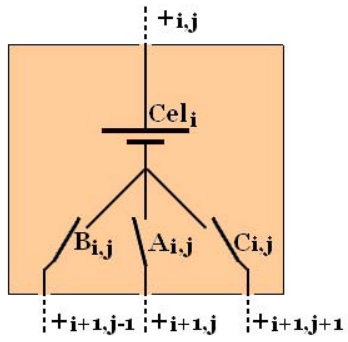

Fig. 10. Module structure for diffusing current in the matrix

From there, it is possible to define in Fig.10 a structure that would allow, through three switches, to apportion the current from one cell to the three adjacent cells into the row below. This module scheme, type $\mathrm{C}-3 \mathrm{C}$ (one cell to three cells) can then be associated in a complete matrix, adapted from the original principle proposed for a matrix because the module are not connected with any other on the row, but only with the previous or the following row. Fig.11 show an example of structure of 3 rows and 3 needed +1 redundant column. This structure implies an effect on the voltage supplied by our structure: it can only be equal to the sum of the voltage of the $n$ cells constituting a column. Indeed, when a cell fails in this case, it is ignored by the system, naturally isolated by the three switches connected to the rest of the matrix. However, for most uses, the voltage delivered by the battery is not a parameter which should be under strict control. 
Often in fact, the voltage delivered by the battery is regulated to the desired value. The constraints on the voltage delivered are rather window constraints: that means the delivered voltage is between a minimum and maximum value.

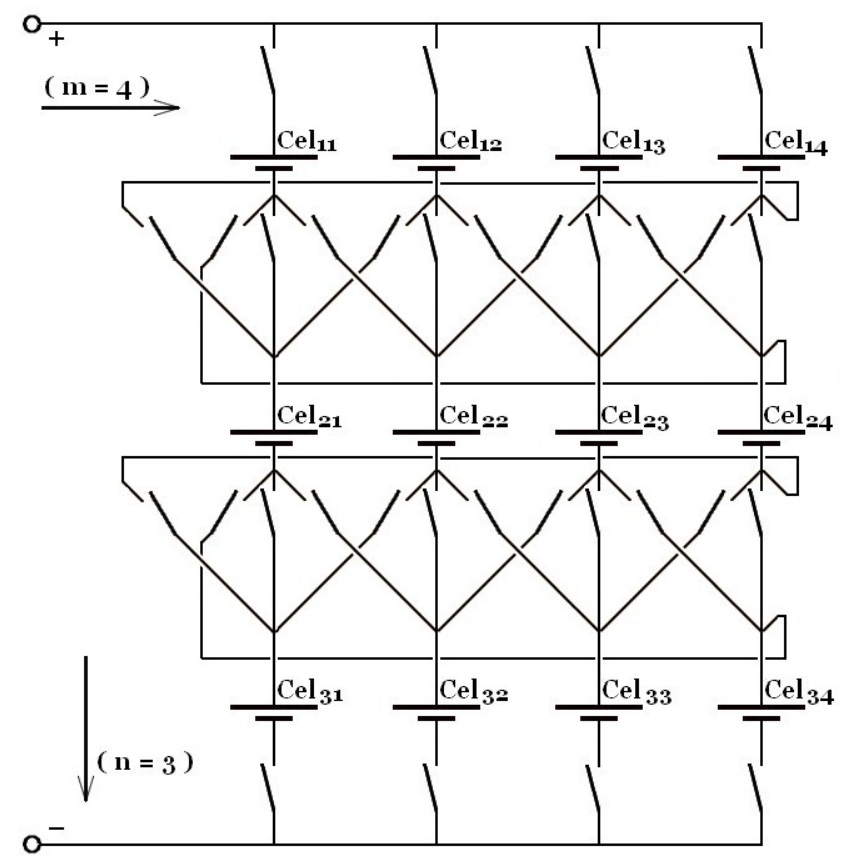

Fig. 11. Example of a C-3C structure supplying an amperage of 3-unit current under a 3-unit tension

( $n=3$ rows; $m=4$ columns, including the redundancy column)

The structure can also continue to be used in degraded mode. That is to say, it continues to provide, under the rated voltage, a current less than the maximum current (which is equal to $(m-1)$ current units) while it remains at least one cell working on in the line that includes the more faulty cells.

\section{RELIABNILITY OF STRUCTURES}

From a Reliability, Availability, Maintainability and Safety (RAMS) point of view, if we want the battery to be able to provide $(m-1)$ current units under $n$ voltage units, it means that we have $n^{*}(m-1)$ cells to be reliable. In a first approach, we will consider the reliability of the switches is much higher than the cells. Thus, we can neglect their influence on the overall reliability. The reliability diagram of a basic matrix structure comprising $n$ rows and $(m-1)$ columns consists of a $n^{*}(m-1)$ cell series string. The overall reliability $\mathrm{R}_{\text {battery }}(\mathrm{t})$ is, if the reliability of cell is $\mathrm{R}_{\text {cell }}$ :

$$
R_{\text {battery }}(t)=R_{\text {cell }}(t)^{n \cdot(m-1) \cdot t}
$$

Where the reliability $R_{\text {cell }}(t)$ is expressed from the failure rate $\lambda_{\text {cell }}(t)$ of the cell. In mature phase, this failure rate is considered constant and equal to $\lambda_{\text {cell. }}$ So $R_{\text {cell }}$ is express in the form :

$$
R_{\text {cell }}(t)=e^{\lambda_{\text {cell }} t}
$$

The first structures (Fig. $7 \mathrm{a}$ and $7 \mathrm{~b}$ ) with $n$ rows and $(m-1)$ columns have an overall reliability equal to formula (2). With our hypothesis, it leads to a $\mathrm{MTTF}_{\text {classical, }}$, which represents the integral of reliability between 0 and infinity, given by equation (4). Indeed, a series connection of a wiring imparts $n$ rows consisting of $(m-1)$ cells in series. A parallel wiring associate $(m-1)$ columns of $n$ rows each. The second and third explain respectively in Fig.7c) and 5, are equivalent in terms of reliability, if we does not associate redundancy cells.

$$
M T T F_{\text {classical }}=\frac{1}{n \cdot(m-1) \cdot \lambda_{\text {cell }}}
$$

Thus, whatever the structure, the reliability is the same. For example, if we consider a small battery consists of 3 rows $(n=3)$ and three columns $((m-1)=3)$, with cells having a failure rate of $10^{-3}$ by unit of time, we get a battery having a MTTF only 111 time units. Each cell used has a MTTF 1000 units of time.

$$
\mathrm{MTTF}_{\text {redundancy }}=\sum_{k=0}^{n \cdot(m-1)}\left(\begin{array}{c}
n \cdot(m-1) \\
k
\end{array}\right) \frac{1}{(n \cdot(m-1))^{k} \cdot \lambda_{\text {cell }}}
$$

If we had classically a redundant cell associated to each elementary cell, the MTTF would have been improved up to 287 , by equation (5) [the reliability of a cell association with a cold redundancy peer don't follow an exponential law]. And if we add only a redundancy column, the MTTF is given by equation (6). In the example, it reaches 148. In order to compare, we consider for equations (5) and (6) that the matrix have $(m-1)$ columns and 1 redundancy.

$$
\begin{aligned}
& \operatorname{MTTF}_{1 \text { column }}=\frac{m}{n \cdot(m-1)^{2} \cdot \lambda_{\text {cell }}} \\
& R_{\text {row }}(t)=e^{-(m-1) \cdot \lambda_{\text {cell }} \cdot t} \cdot\left(1+(m-1) \cdot \lambda_{\text {cell }} \cdot t\right)
\end{aligned}
$$

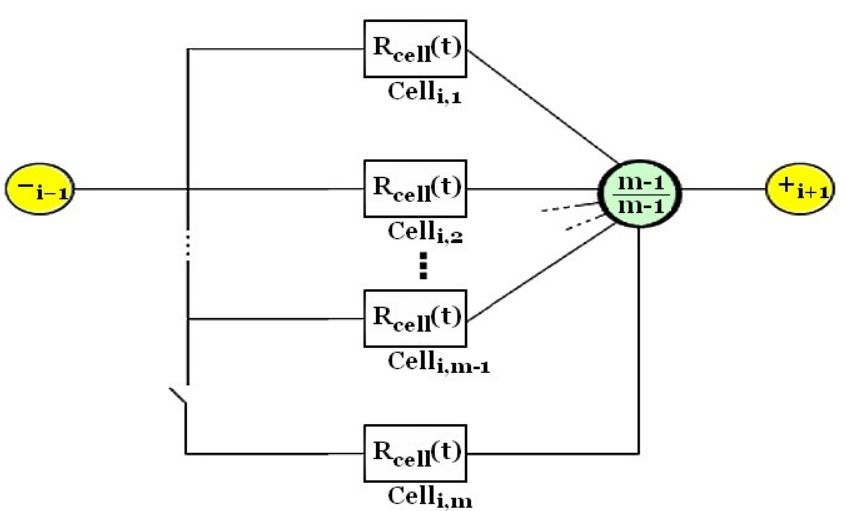

Fig.12. Reliability diagram of a C-3C row: a system with (m-1) operating unit and 1 standby unit, and perfect changeover

Our C-3C structure provides some improvement in reliability. In fact, if we reason by row, we can determine the reliability from the reliability diagram in Fig.12. The reliability of one row $R_{\text {row }}$ is done in equation (7). Then, the overall reliability $\mathrm{R}_{\mathrm{c}-3 \mathrm{c}}$ of our structure, with one - and only 
one - redundant column is given by the $\mathrm{R}_{\text {row }}$ in power $n$ (number of row). So, we found a better MTTF of the system with $n$ row and $m$ column than the classic, as:

$$
\operatorname{MTTF}_{C-3 \mathrm{C}}=\sum_{k=0}^{n}\left(\begin{array}{l}
n \\
k
\end{array}\right) \frac{1}{n^{k+1} \cdot(m-1) \cdot \lambda_{\text {cell }}}
$$

Time to failure increases up to 263 units of time by equation (8), with the $\mathrm{C}-3 \mathrm{C}$ device; not as much as with the maximum redundancy, but much better than with a single column redundancy. Some examples of structures according to the number of columns and rows are provided in Table.1. By using the same number of redundant cells within the assembly having a redundant column, the structure $\mathrm{C}-3 \mathrm{C}$ improves the battery $\mathrm{MTTF}_{\text {battery. }}$

\begin{tabular}{|c|c|c|c|c|c|}
\hline \multicolumn{2}{|c|}{$\boldsymbol{\lambda = 0 , 0 0 1}$} & \multicolumn{4}{c|}{ MTTF } \\
\hline m-1 & $\mathbf{n}$ & classical & redundancy & $\mathbf{1}$ column & C-3C \\
\hline 1 & 1 & 1000 & 2000 & 2000 & - \\
\hline \multirow{4}{*}{3} & 1 & 333 & 790 & 667 & 667 \\
\cline { 2 - 7 } & 2 & 167 & 420 & 333 & 375 \\
\cline { 2 - 7 } & 3 & 111 & 289 & 222 & 263 \\
\cline { 2 - 7 } & 4 & 83 & 218 & 167 & 203 \\
\cline { 2 - 7 } & 10 & 33 & 89 & 67 & 86 \\
\hline \multirow{4}{*}{10} & 1 & 100 & 259 & 100 & 200 \\
\cline { 2 - 6 } & 2 & 50 & 133 & 67 & 113 \\
\cline { 2 - 6 } & 3 & 33 & 89 & 50 & 79 \\
\cline { 2 - 6 } & 4 & 25 & 67 & 40 & 61 \\
\hline
\end{tabular}

Table 1) Comparison of the MTTF of matrix structures with identical cell $\lambda_{\text {cell }}=10^{-3}$ defect by unit of time

Our scheme is very relevant in the matrices of significant size. As soon as the number of rows and/or the number of columns increases, the $\mathrm{C} 3 \mathrm{C}$ profits redundancy: with respect to a redundancy column, using the same number of cells, the MTTF is improved $50 \%$ in the case where $n=1$ and $m=3$, this improvement amounts to more than $235 \%$ when the matrix is 10 to 10 .

\section{CONCLUSION}

There are many technological solutions to meet the demand to provide ( $m$ - 1$)$ current units under $n$ voltage units for a battery. Most operational structures present internal redundancy to meet this demand. Also, they have many switches with the aim of isolating the faulty cells and thereby preserve those with low SoC can no longer provide power without being deteriorated.

The C-3C structure presented here allows limiting redundancy to a minimum while improving the performance in terms of reliability. It also allows offering a large number of possible internal configurations, fostering the overall performance of a battery, by the multiplicity of possible paths for the current [11]. On the other hand, it could well stand as an easily implantable alternative to enable dynamic reconfiguration [12] while also allowing good fault tolerance.

We showed that it is possible to obtain the same overall reliability of the battery with the same level of redundancy that addind an extra column to the matrix. This improvement, however, is lower than if all cells were doubled for redundancy. On the other hand, the number of transistors required is two to three times more important (depending on the combinaison of $n$ rows and $m$ columns).

\section{REFERENCES}

[1] J. Kim et B.-H. Cho, « State-of-Charge Estimation and State-of-Health Prediction of a Li-Ion Degraded Battery Based on an EKF Combined With a Per-Unit System », IEEE Trans. Veh. Technol., vol. 60, nº 9, p. 4249-4260, nov. 2011.

[2] Y.-S. Lee et M.-W. Cheng, « Intelligent control battery equalization for series connected lithium-ion battery strings », IEEE Trans. Ind. Electron., vol. 52, n ${ }^{\circ}$, p. 1297-1307, oct. 2005.

[3] T. Kim, W. Qiao, L. Qu, « A Series-Connected Self-Reconfigurable Multicell Battery Capable of Safe and Effective Charging/Discharging and Balancing Operations 》, Applied Power Electronics Conference and Exposition (APEC), 2012 Twenty-Seventh Annual IEEE, 2012.

[4] F. Baronti, G. Fantechi, R. Roncella, et R. Saletti, « Design of a module switch for battery pack reconfiguration in high-power applications $\gg$, 2012 IEEE International Symposium on Industrial Electronics (ISIE), 2012, p. 1330-1335.

[5] T. Kim, W. Qiao, et L. Qu, « Series-connected self-reconfigurable multicell battery 》, 2011 Twenty-Sixth Annual IEEE Applied Power Electronics Conference and Exposition (APEC), 2011, p. 13821387.

[6] L. He, L; Kong, S. Lin, S. Ying, Y. Gu, Y. He, C. Liu, «Reconfiguration-Assisted Charging in Large-Scale Lithium-ion Battery Systems $\gg$, ICCPS'14, April 14-17 2014, Berlin, Germany, 2013.

[7] V.R.H. Lorentz, M.M. Wenger, J.L. Grosch, M. Giegerich and all., "Novel Cost-Efficient Contactless Distributed Monitoring Concept for Smart Battery Cells". 2012 IEEE International Symposium on Industrial Electronics (ISIE), 2012, p. 1342-1347

[8] Hahnsang Kim, Kang G. Shin, Dependable, efficient, scalable architecture for management of large-scale batteries, 1st ACM/IEEE International Conference on Cyber-Physical Systems Pages 178-187, 2010.

[9] H. Kim et K. G. Shin, « Scheduling of Battery Charge, Discharge, and Rest », 30th IEEE Real-Time Systems Symposium, 2009, RTSS 2009, 2009, p. 13-22.

[10] F. Jin et K. G. Shin, « Pack Sizing and Reconfiguration for Management of Large-Scale Batteries », présenté à 2012 IEEE/ACM Third International Conference on Cyber-Physical Systems (ICCPS), 2012, p. 138-147.

[11] L. Cloth, M. R. Jongerden, et B. R. Haverkort, « Computing Battery Lifetime Distributions », 37th Annual IEEE/IFIP International Conference on Dependable Systems and Networks, 2007. DSN '07, 2007, p. 780-789.

[12] S. Ci, J. Zhang, H. Sharif, et M. Alahmad, « Dynamic reconfigurable multi-cell battery: A novel approach to improve battery performance », 2012 Twenty-Seventh Annual IEEE Applied Power Electronics Conference and Exposition (APEC), 2012, p. 439-442

We thaznk the Lyon draduate school of engineering Electronics Electrotechniocal Automatic ED160 for its financial support. 